\title{
COMMENTARY
}

\section{Biomarkers of focal and diffuse traumatic brain injury}

\author{
Pieter E Vos* \\ See related research by Mondello et al., http://ccforum.com/content/15/3/R156
}

\begin{abstract}
Traumatic brain injury (TBI) is a pathologically heterogeneous disease affecting people of all ages. The highest incidence of TBI occurs in young people and the average age is 30 to 40 years. Injury grading may range from mild with a low frequency ( 1 per 100) of life-threatening intracranial hematoma that needs immediate neurosurgical operation and very low mortality $(1$ per 1,000$)$ to severe with a high likelihood of life-threatening intracranial hematoma (up to 1 per 3), a 40\% case fatality rate and a high disability rate (2 per 3 ) in survivors. Estimation of the prognosis in severe TBI is currently based on demographic and clinical predictors, including age, Glasgow Coma Scale, pupillary reactions, extracranial injury (hypotension and hypoxia) and computed tomography indices (brain swelling, focal mass lesions, subarachnoid hemorrhage). Biomarkers reflecting damage to neurons and astrocytes may add important complementary information to clinical predictors of outcome and provide insight into the pathophysiology of TBI.
\end{abstract}

In the previous issue of Critical Care, Mondello and colleagues [1] demonstrate that easy to obtain biochemical markers of brain injury in peripheral blood may complement current predictors of outcome in severe traumatic brain injury (TBI). The study also advocates that use of biomarkers may contribute to better understanding of the pathophysiological mechanisms of brain damage. After TBI, brain tissue-specific neuronal and glial proteins may suddenly appear in the systemic circulation via leakage through the disturbed blood brain barrier or via leakage to the cerebrospinal fluid and subsequent

*Correspondence: p.vos@neuro.umcn.nl

Department of Neurology, Radboud University Nijmegen Medical Centre, 6500 HB, Nijmegen, The Netherlands normal transport to the circulation. Despite the strength of the association between existing predictors of outcome, the prognostic value is still modest. Studies in more than 10,000 patients demonstrate a diagnostic accuracy of 0.70 to 0.84 , which is insufficient as a diagnostic and prognostic tool in individual patients $[2,3]$. Current models explain only $35 \%$ of the outcome variation [4]. Considering the large proportion of outcome variation that remains unexplained, the models need to be refined [5].

Mondello and colleagues determined in a cohort of 81 severe TBI patients the time course of neuronal (ubiquitin carboxy-terminal hydrolase (UCH)-L1) and astroglial (glial fibrillary acidic protein (GFAP)) biomarker levels in serum in the first 24 hours after severe focal and diffuse injuries. UCH-L1 and GFAP were selected because of abundant expression in neurons (UCH-L1) and astrocytes (GFAP). UCH-L1 is involved in the addition or removal of ubiquitin from abnormal proteins destined for proteasomal degradation and has previously been used as a neuronal cell soma marker [6]. Initial results in cerebrospinal fluid showed higher levels of UCH-L1 in patients with low Glasgow Coma Scale (GCS) scores, post-injury complications, early mortality and poor 6-month outcome. GFAP was chosen because of its high specificity for central nervous system astrocytes and its strong association with GCS, injury severity, intracranial pathology and outcome after TBI $[7,8]$.

Interestingly, the study shows markedly increased serum levels for UCH-L1 (11.3-fold) and GFAP (28.7-fold) in the first 24 hours post-injury compared to controls. Using the intracranial diagnosis of the Trauma Coma Data Bank classification, different pathways for UCH-L1 and GFAP were found; UCH-L1 levels were high in diffuse injuries and GFAP serum levels were particularly increased in the presence of focal mass lesions. In the prediction of outcome, the combination of UCH-L1, age and GCS score predicted death at 6 months post-injury with an accuracy of $94 \%$, although the combination of GFAP and age was equally predictive. This corroborates recent findings demonstrating that GFAP serum levels highly correlate with the presence of focal lesions on computed tomography $(r=0.73)$ [7]. Multivariate modeling to 
predict mortality yielded diagnostic accuracies of 0.92 for the combination of GFAP with pupillary reactions and 0.92 for the combination of GFAP with mass lesions.

The study by Mondello and colleagues focused on focal versus diffuse injuries defined by computed tomography rather than magnetic resonance imaging. This dichotomization did not allow the levels of biomarkers in patients with mixed focal and diffuse pathology to be studied. Recent magnetic resonance imaging demonstrates that focal and diffuse injuries occur separately but also coexist in moderate/severe TBI. Pure forms of focal injury occur in $28 \%$ of moderate/severe TBI cases, pure diffuse axonal injury in $22 \%$, while mixed focal and diffuse injuries occur in 50\% [9]. The cohort size of 81 in this transatlantic study in four centers limits the number of potential predictors that could be assessed and only three (age, $\mathrm{UCH}-\mathrm{L} 1, \mathrm{GCS})$ were included in the final model to predict mortality.

The investigation did not obtain other outcome measures (global or cognitive) than mortality (in hospital and at 6 months). Knowing that a biomarker predicts mortality, however, is important when treatment limitation or withdrawal is considered. In this study UCH-L1 together with age and the GCS were included in the final model, suggesting that biomarker test results are specific for a particular outcome category (death) and may increase the confidence with which final decisions can be made.

More importantly, however, will biomarkers ultimately add to the prediction of long-term outcome of survivors to answer whether a patient will reach independency from care givers or return to work? In this respect it is promising that different results were obtained for the two markers with diffuse and focal injuries. The importance of the pathological heterogeneity of focal and diffuse TBI lies in the fact that patients with the same GCS score can have markedly different treatments, recovery patterns and outcomes depending on the causative lesion.

A challenging next step is to obtain cutoff levels for neuronal and glial markers of TBI with small falsepositive rates not only for death but also for global unfavorable outcome measures such as disability or cognitive dysfunction. To achieve this goal, larger (multicenter) studies are needed to establish precise threshold values and to demonstrate that information obtained from biomarkers in addition to clinical variables indeed allows for sufficiently accurate outcome prediction in individual patients. In addition, it will be interesting if prolonged determination of biomarker levels in the acute phase can be associated with secondary complications such as increased intracranial pressure, decreased cerebral perfusion pressure and effects of treatment.

\section{Abbreviations}

GCS, Glasgow Coma Scale; GFAP, glial fibrillary acidic protein; TBI, traumatic brain injury; $\mathrm{UCH}$, ubiquitin carboxy-terminal hydrolase.

\section{Competing interests}

PEV serves on a scientific advisory board and received funding for travel and speaker honoraria from Ever Neuro Pharma; serves as a member of the editorial advisory board of The European Journal of Neurology; and has received honoraria for lectures or educational activities not funded by the industry.

Published: 18 August 2011

\section{References}

1. Mondello S, Papa L, Buki A, Bullock R, Czeiter E, Tortella F, Wang KK, Hayes RL: Neuronal and glial markers are differently associated with computed tomography findings and outcome in patients with severe traumatic brain injury: a case control study. Crit Care 2011, 15:R156.

2. Steyerberg EW, Mushkudiani N, Perel P, Butcher I, Lu J, McHugh GS, Murray GD, Marmarou A, Roberts I, Habbema JD, Maas Al: Predicting outcome after traumatic brain injury: development and international validation of prognostic scores based on admission characteristics. PLOS Med 2008, 5:e165.

3. MRC CRASH Trial Collaborators, Perel P, Arango M, Clayton T, Edwards P, Komolafe E, Poccock S, Roberts I, Shakur H, Steyerberg E, Yutthakasemsunt S: Predicting outcome after traumatic brain injury: practical prognostic models based on large cohort of international patients. BMJ 2008 336:425-429.

4. Lingsma HF, Roozenbeek B, Steyerberg EW, Murray GD, Maas Al: Early prognosis in traumatic brain injury: from prophecies to predictions. Lancet Neurol 2010, 9:543-554

5. Saatman KE, Duhaime AC, Bullock R, Maas Al, Valadka A, Manley GT: Classification of traumatic brain injury for targeted therapies. J Neurotrauma 2008, 25:719-738.

6. Tongaonkar P, Chen L, Lambertson D, Ko B, Madura K: Evidence for an interaction between ubiquitin-conjugating enzymes and the $26 \mathrm{~S}$ proteasome. Mol Cell Biol 2000, 20:4691-4698.

7. Vos PE, Jacobs B, Andriessen TM, Lamers KJ, Borm GF, Beems T, Edwards M, Rosmalen CF, Vissers JL: GFAP and S100B are biomarkers of traumatic brain injury: an observational cohort study. Neurology 2010, 75:1786-1793.

8. Pelinka LE, Kroepfl A, Schmidhammer R, Krenn M, Buchinger W, Redl H, Raabe A: Glial fibrillary acidic protein in serum after traumatic brain injury and multiple trauma. J Trauma 2004, 57:1006-1012.

9. Skandsen T, Kvistad KA, Solheim O, Strand IH, Folvik M, Vik A: Prevalence and impact of diffuse axonal injury in patients with moderate and severe head injury: a cohort study of early magnetic resonance imaging findings and 1-year outcome. J Neurosurg 2010, 113:556-563.

doi:10.1186/cc10290

Cite this article as: VOS PE: Biomarkers of focal and diffuse traumatic brain injury. Critical Care 2011, 15:183. 